\title{
Improved lumped models for transient combined convective and radiative cooling of multi-layer composite slabs
}

\author{
Chen $\mathrm{An}^{\mathrm{a}}$, Jian $\mathrm{Su}^{\mathrm{b}, *}$ \\ a Ocean Engineering Program, COPPE, Universidade Federal do Rio de Janeiro, CP 68508, Rio de Janeiro, 21941-972, Brazil \\ ${ }^{\mathrm{b}}$ Nuclear Engineering Program, COPPE, Universidade Federal do Rio de Janeiro, CP 68509, Rio de Janeiro, 21941-972, Brazil
}

\section{A R T I C L E I N F O}

Article history:

Received 25 January 2011

Accepted 10 April 2011

Available online 16 April 2011

\section{Keywords:}

Transient heat conduction

Lumped model

Multi-layer composite slabs

Radiative cooling

Convective cooling

Wall heat transfer

\begin{abstract}
A B S T R A C T
Improved lumped parameter models were developed for the transient heat conduction in multi-layer composite slabs subjected to combined convective and radiative cooling. The improved lumped models were obtained through two-point Hermite approximations for integrals. Transient combined convective and radiative cooling of three-layer composite slabs was analyzed to illustrate the applicability of the proposed lumped models, with respect to different values of the Biot numbers, the radiationconduction parameter, the dimensionless thermal contact resistances, the dimensionless thickness, and the dimensionless thermal conductivity. It was shown by comparison with numerical solution of the original distributed parameter model that the higher order lumped model $\left(H_{1,1} / H_{0,0}\right.$ approximation) yielded significant improvement of average temperature prediction over the classical lumped model. In addition, the higher order $\left(H_{1,1} / H_{0,0}\right)$ model was applied to analyze the transient heat conduction problem of steel-concrete-steel sandwich plates.
\end{abstract}

(c) 2011 Elsevier Ltd. All rights reserved.

\section{Introduction}

Transient heat conduction problem in multi-layer composite slabs has been extensively studied due to its applications in various technological areas such as dynamical thermal behavior of walls, working regime of heat treatment furnace, thermally protected structures, transient aerodynamic heating of airborne vehicles, and heat transfer in insulation materials [1-8]. Many analytical and numerical approaches have been proposed to obtain the transient response of one-dimensional multi-layer composite conducting slabs with convective cooling boundary conditions. De Monte $[9,10]$ reviewed a historical bibliography and presented a 'natural' analytic approach simplifying the eigenvalues solving process by placing the thermal diffusivity on the time-dependent function side in the form of separated variables. Blanc and Touratier [5] presented a constrained discrete layer model as an approximated solution, in which the interface conditions for heat flux are incorporated into the governing equation of each layer in order to reduce the number of unknowns. Chen and Wang [11,12] estimated the heat gain/loss through building multi-layer constructions using a frequency-domain regression method based on the theoretical frequency-response characteristics of the walls. Oturanç and Sahin [6] solved the transient heat conduction problem in two-layer

\footnotetext{
* Corresponding author. Tel.: +55 212562 8448; fax: +55 2125628444 .

E-mail addresses: chen@lts.coppe.ufrj.br (C. An), sujian@ufrj.br (J. Su).
}

composite wall analytically using spectral analysis, where an asymptotic formula is derived for the eigenvalues of the spectral problem. Antonopoulos et al. [13] applied orthogonal expansion of functions over multi-layer walls to obtain an analytical solution for on-site estimation of the layer thermal properties of multi-layer walls, referred to as the inverse wall heat conduction problem. Based on finite difference method, Charette et al. [4] and Asan [1] analyzed the heat conduction problem in a composite medium with variable thermal properties and investigated the optimum insulation position from maximum time lag and minimum decrement factor point of view, respectively.

The analysis of transient conduction of multi-layer systems is more complicated when radiative cooling or heating at the boundaries is considered. Sundén [14] presented numerical solutions based on finite difference method of the thermal response of a composite slab subjected to a time-varying incident heat flux on one side and combined convective and radiative cooling on the other side. Miller and Weaver [15] developed an analytical model based on separation of variables technique to predict the temperature distribution through a multi-layer composite structure subjected to combined convection and linearized radiation boundary conditions. To predict the effective thermal conductivities for multi-layer thermal insulations for high temperature fuel cell applications, Spinnler et al. [7] performed experimental study using a new design of guarded hot plate apparatus and solved the combined conduction and radiation heat transfer problem theoretically based on the 


\begin{tabular}{|c|c|c|c|}
\hline \multicolumn{2}{|c|}{ Nomenclature } & \multirow{3}{*}{$\begin{array}{l}x_{i} \\
x_{M+1}\end{array}$} & \multirow{3}{*}{$\begin{array}{l}\text { values of the space coordinate at the inner boundary } \\
\text { surfaces } \\
\text { value of the space coordinate at the right-side } \\
\text { boundary surface }\end{array}$} \\
\hline$B i_{1}$ & Biot number at the left-side boundary surface & & \\
\hline$B i_{2}$ & Biot number at the right-side boundary surface & & \\
\hline$c_{p i}$ & specific heat for the $i$-th layer & & \\
\hline$h_{1}$ & convective heat transfer coefficient at the left-side & \multicolumn{2}{|c|}{ Greek Letters } \\
\hline & boundary surface & $\alpha_{i}$ & thermal diffusivity for the $i$-th layer \\
\hline$h_{2}$ & $\begin{array}{l}\text { convective heat transfer coefficient at the right-side } \\
\text { boundary surface }\end{array}$ & $\begin{array}{l}\alpha_{r e f} \\
\delta_{i}\end{array}$ & $\begin{array}{l}\text { reference thermal diffusivity } \\
\text { dimensionless thickness for the } i \text {-th layer }\end{array}$ \\
\hline$k_{i}$ & thermal conductivity for the $i$-th layer & $\epsilon$ & surface emissivity \\
\hline$k_{\text {ref }}$ & reference thermal conductivity & $\eta$ & dimensionless space coordinate \\
\hline $\begin{array}{l}L \\
M\end{array}$ & $\begin{array}{l}\text { total thickness of multi-layer composite slabs } \\
\text { number of layers }\end{array}$ & $\eta_{1}$ & $\begin{array}{l}\text { value of the dimensionless space coordinate at the left- } \\
\text { side boundary surface }\end{array}$ \\
\hline $\begin{array}{l}N_{r c} \\
R c_{j}\end{array}$ & $\begin{array}{l}\text { radiation-conduction parameter } \\
\text { thermal contact resistances at the inner boundary }\end{array}$ & $\eta_{i}$ & $\begin{array}{l}\text { values of the dimensionless space coordinate at the } \\
\text { inner boundary surfaces }\end{array}$ \\
\hline \multirow{2}{*}{$R c_{i}^{*}$} & $\begin{array}{l}\text { surfaces } \\
\text { dimensionless thermal contact resistances at the inner }\end{array}$ & $\eta_{M+1}$ & $\begin{array}{l}\text { value of the dimensionless space coordinate at the } \\
\text { right-side boundary surface }\end{array}$ \\
\hline & boundary surfaces & $\theta_{a}$ & dimensionless adiabatic surface temperature \\
\hline$t$ & time & $\theta_{i}$ & dimensionless temperature for the $i$-th layer \\
\hline$T_{0}$ & $\begin{array}{l}\text { uniform initial temperature of multi-layer composite } \\
\text { slabs }\end{array}$ & $\theta_{m}$ & $\begin{array}{l}\text { dimensionless left-side environmental fluid } \\
\text { temperature }\end{array}$ \\
\hline$T_{a}$ & adiabatic surface temperature & $\kappa_{i}$ & dimensionless thermal diffusivity for the $i$-th layer \\
\hline$T_{f}$ & right-side environmental fluid temperature & $\lambda_{i}$ & dimensionless thermal conductivity for the $i$-th layer \\
\hline$T_{i}$ & temperature for the $i$-th layer & $\rho_{i}$ & density for the $i$-th layer \\
\hline$T_{m}$ & left-side environmental fluid temperature & $\sigma$ & Stefan-Boltzmann constant \\
\hline$T_{s}$ & radiation sink temperature & $\tau$ & dimensionless time \\
\hline$x$ & space coordinate & & \\
\hline \multirow[t]{2}{*}{$x_{1}$} & value of the space coordinate at the left-side boundary & \multicolumn{2}{|c|}{ Subscripts } \\
\hline & surface & $\begin{array}{l}i \\
a v i\end{array}$ & $\begin{array}{l}i \text {-th layer defined in the domain } \\
\text { average for the } i \text {-th layer }\end{array}$ \\
\hline
\end{tabular}

energy conservation equation approach together with a scaling model. Besides, Li and Cheng [8] applied energy balance equation to obtain a mathematical model for the combined radiation and conduction heat transfer in multi-layer perforated insulation material using in space, which was solved numerically by iterative method combining with the alternating directions implicit method.

The lumped parameter approach has been widely used to analyze the dynamical thermal behavior of structures [16-21]. A simplified formulation of the transient heat conduction extremely useful and sometimes even mandatory as in the analysis of other complex thermal systems. Due to the inherent limitation of the classical lumped parameter approach, improved lumped models have been developed by different approaches [22-27]. Cotta and Mikhailov [22] proposed a systematic formalism to provide improved lumped parameter formulation for steady and transient heat conduction problems based on Hermite approximation for integrals that define averaged temperatures and heat fluxes. This approach has been shown to be efficient in a great variety of practical applications [28-32]. In this work, we present improved lumped models for transient combined convective and radiative cooling of multi-layer composite slabs, extending previous works on the particular cases of a single slab subjected to convective cooling [23], radiative cooling [24], and combined convectiveradiative cooling [32]. The proposed lumped models are obtained through two-point Hermite approximations for integrals [33,22]. By comparing with numerical solution of the original distributed parameter formulation, it is shown that the higher order improved lumped model $\left(H_{1,1} / H_{0,0}\right.$ approximation) yields significant improvement of average temperature prediction over the classical lumped model. In addition, the higher order $\left(H_{1,1} / H_{0,0}\right)$ model is applied to analyze the transient heat conduction problem of steelconcrete-steel sandwich plates.

\section{The mathematical formulation}

Consider one-dimensional transient heat conduction in multilayer composite slabs consisting of $M$ parallel layers in cartesian coordinates, subjected to convective heat transfer at the left side and combined convective and radiative heat transfer at the right side, as shown in Fig. 1. The layers are labeled as 1 to $M$ from the left to right. Let $x$ be the coordinate perpendicular to the layers, $x_{i}, i=1,2, \ldots, M$, represent its value at the left surface of each

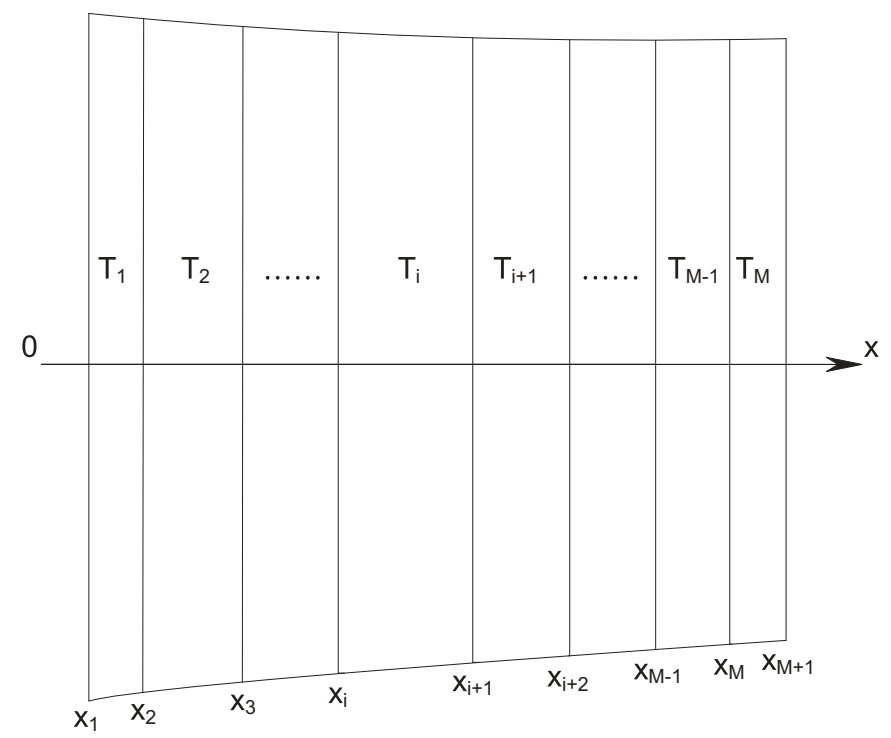

Fig. 1. Illustration of M-layer composite slabs. 
layer, and $x_{M+1}$ be the coordinate value at the right surface of $M$-th layer. It is assumed that the thermophysical properties of the layers are homogeneous, isotropic and independent of the temperature. There is no volumetric heat generation in the slabs. The thermal contact resistance between adjacent $i$-th and $(i+1)$-th layer at interface $x_{i+1}, i=1,2, \ldots, M-1$, is represented by $R c_{i}$. Initially, all the layers are at a specified uniform temperature $T_{0}$. At $t=0$, the composite is exposed to an environment of a constant fluid temperature $T_{m}$ with a constant convective heat transfer coefficient $h_{1}$ at the left side, and an environment of a constant fluid temperature $T_{f}$ with a constant heat transfer coefficient $h_{2}$ and a constant radiation sink temperature $T_{S}$ at the right side.

The mathematical formulation of the one-dimensional transient heat conduction problem is given by

$\frac{\partial T_{i}}{\partial t}=\alpha_{i} \frac{\partial^{2} T_{i}}{\partial x^{2}}$, in $x_{i}<x<x_{i+1}, \quad i=1,2, \ldots, M$, for $t>0$,

with the following boundary and interface conditions

$-k_{1} \frac{\partial T_{1}}{\partial x}=h_{1}\left(T_{m}-T_{1}\right), \quad$ at $x=x_{1}$, for $t>0$,

$-k_{M} \frac{\partial T_{M}}{\partial x}=h_{2}\left(T_{M}-T_{f}\right)+\epsilon \sigma\left(T_{M}^{4}-T_{s}^{4}\right)$, at $x=x_{M+1}$, for $t>0$,

$-k_{i} \frac{\partial T_{i}}{\partial x}=\frac{T_{i}-T_{i+1}}{R c_{i}}$, at $x=x_{i+1}, i=1,2, \ldots, M-1$, for $t>0$,

$k_{i} \frac{\partial T_{i}}{\partial x}=k_{i+1} \frac{\partial T_{i+1}}{\partial x}$, at $x=x_{i+1}, i=1,2, \ldots, M-1$, for $t>0$,

and the initial conditions for each layer

$T_{i}(x, 0)=T_{0}, \quad$ in $x_{i}<x<x_{i+1}, \quad i=1,2, \ldots, M$, at $t=0$,

where $T_{i}(x, t)$ is the temperature in the $i$-th layer, $t$ the time, $\alpha_{i}\left(=k_{i} / \rho_{i} c_{p i}\right)$ the thermal diffusivity, $k_{i}$ the thermal conductivity, $\rho_{i}$ the density, $c_{p i}$ the specific heat, $\epsilon$ the surface emissivity, and $\sigma$ the Stefan-Boltzmann constant.

It should be noted that in general the environmental fluid temperature $T_{f}$ differs from the radiation sink temperature $T_{s}$. It is

Table 1

Comparison of lumped models against finite difference solution for dimensionless average temperatures $\theta_{a v 1}(\tau), \theta_{a v 2}(\tau)$ and $\theta_{a v 3}(\tau)$ at the different dimensionless time for Case I-1 $\left(N_{r c}=8\right)$.

\begin{tabular}{lllll}
\hline$\tau$ & FD solution & CLSA & $H_{0,0} / H_{0,0}$ & $H_{1,1} / H_{0,0}$ \\
\hline$\theta_{a v 1}(\tau)$ & & & & \\
0.01 & 0.977077 & 0.977009 & 0.977835 & 0.977556 \\
0.05 & 0.898266 & 0.893692 & 0.910351 & 0.903590 \\
0.10 & 0.817321 & 0.798962 & 0.837296 & 0.824230 \\
0.20 & 0.678372 & 0.632965 & 0.696711 & 0.682392 \\
0.50 & 0.380030 & 0.313717 & 0.380668 & 0.379461 \\
1.00 & 0.158777 & 0.118866 & 0.153442 & 0.157714 \\
$\theta_{a v 2}(\tau)$ & & & & \\
0.01 & 0.996140 & 0.993741 & 0.996449 & 0.996593 \\
0.05 & 0.937442 & 0.919931 & 0.929336 & 0.938978 \\
0.10 & 0.843144 & 0.809985 & 0.824080 & 0.844829 \\
0.20 & 0.675684 & 0.624875 & 0.647446 & 0.677444 \\
0.50 & 0.365143 & 0.304330 & 0.340116 & 0.365632 \\
1.00 & 0.153164 & 0.116282 & 0.140259 & 0.152645 \\
$\theta_{a v 3}(\tau)$ & & & & \\
0.01 & 0.716278 & 0.681148 & 0.708231 & 0.716658 \\
0.05 & 0.306139 & 0.287994 & 0.300078 & 0.302117 \\
0.10 & 0.181484 & 0.184685 & 0.182581 & 0.179309 \\
0.20 & 0.130650 & 0.139459 & 0.133520 & 0.131164 \\
0.50 & 0.089373 & 0.089328 & 0.089214 & 0.089909 \\
1.00 & 0.062863 & 0.060252 & 0.062170 & 0.062962 \\
\hline
\end{tabular}

convenient to introduce the adiabatic surface temperature $T_{a}$, defined by

$h_{2}\left(T_{a}-T_{f}\right)+\epsilon \sigma\left(T_{a}^{4}-T_{s}^{4}\right)=0$

The boundary condition Eq. (3) can be rewritten with use of the adiabatic surface temperature

$-k_{M} \frac{\partial T_{M}}{\partial x}=h_{2}\left(T_{M}-T_{a}\right)+\epsilon \sigma\left(T_{M}^{4}-T_{a}^{4}\right)$, at $x=x_{M+1}$, for $t>0$,

The mathematical formulation given by the system of Eqs. $(1,2,4-6,8)$ can now be expressed in dimensionless form as follows:

$\frac{\partial \theta_{i}}{\partial \tau}=\kappa_{i} \frac{\partial^{2} \theta_{i}}{\partial \eta^{2}}$, in $\eta_{i}<\eta<\eta_{i+1}, \quad i=1,2, \ldots, M$, for $\tau>0$,

$-\lambda_{1} \frac{\partial \theta_{1}}{\partial \eta}=B i_{1}\left(\theta_{m}-\theta_{1}\right), \quad$ at $\quad \eta=\eta_{1}, \quad$ for $\tau>0$,

$-\lambda_{M} \frac{\partial \theta_{M}}{\partial \eta}=B i_{2}\left(\theta_{M}-\theta_{a}\right)+N_{r c}\left(\theta_{M}^{4}-\theta_{a}^{4}\right)$, at $\eta=\eta_{M+1}$, for $\tau>0$,

$-\lambda_{i} \frac{\partial \theta_{i}}{\partial \eta}=\frac{\theta_{i}-\theta_{i+1}}{R c_{i}^{*}}$, at $\eta=\eta_{i+1}, i=1,2, \ldots, M-1$, for $\tau>0$,

$\lambda_{i} \frac{\partial \theta_{i}}{\partial \eta}=\lambda_{i+1} \frac{\partial \theta_{i+1}}{\partial \eta}$, at $\eta=\eta_{i+1}, i=1,2, \ldots, M-1$, for $\tau>0$

$\theta_{i}(\eta, 0)=1$, in $\eta_{i}<\eta<\eta_{i+1}, i=1,2, \ldots, M$, at $\tau=0$,

where the dimensionless parameters are defined by

$\theta_{i}=\frac{T_{i}}{T_{0}}, \quad \eta=\frac{x}{L}, \quad \eta_{i}=\frac{x_{i}}{L}, \quad \tau=\frac{\alpha_{r e f} t}{L^{2}}$,

$\kappa_{i}=\frac{\alpha_{i}}{\alpha_{\text {ref }}}, \quad \lambda_{i}=\frac{k_{i}}{k_{\text {ref }}}, \quad R c_{i}^{*}=\frac{R c_{i} k_{\text {ref }}}{L}$,

$B i_{1}=\frac{h_{1} L}{k_{\text {ref }}}, \quad B i_{2}=\frac{h_{2} L}{k_{\text {ref }}}, \quad N_{r c}=\frac{\epsilon \sigma L T_{0}^{3}}{k_{\text {ref }}}$.

$(15 \mathrm{~h}-\mathrm{j})$

Table 2

Comparison of lumped models against finite difference solution for dimensionless average temperatures $\theta_{a v 1}(\tau), \theta_{a v 2}(\tau)$ and $\theta_{a v 3}(\tau)$ at the different dimensionless time for Case I-2 $\left(N_{r c}=32\right)$.

\begin{tabular}{lllll}
\hline$\tau$ & FD Solution & CLSA & $H_{0,0} / H_{0,0}$ & $H_{1,1} / H_{0,0}$ \\
\hline$\theta_{a v 1}(\tau)$ & & & & \\
0.01 & 0.977077 & 0.976943 & 0.978254 & 0.977785 \\
0.05 & 0.898204 & 0.892134 & 0.911842 & 0.904224 \\
0.10 & 0.816621 & 0.795372 & 0.837165 & 0.823686 \\
0.20 & 0.676158 & 0.627851 & 0.694092 & 0.680044 \\
0.50 & 0.377791 & 0.310529 & 0.378190 & 0.377199 \\
1.00 & 0.157964 & 0.117991 & 0.152610 & 0.156905 \\
$\theta_{a v 2}(\tau)$ & & & & \\
0.01 & 0.994634 & 0.990535 & 0.994885 & 0.995275 \\
0.05 & 0.929677 & 0.908304 & 0.920166 & 0.931360 \\
0.10 & 0.834630 & 0.798228 & 0.814498 & 0.836428 \\
0.20 & 0.669535 & 0.616660 & 0.641059 & 0.671385 \\
0.50 & 0.362661 & 0.301079 & 0.337694 & 0.363153 \\
1.00 & 0.152380 & 0.115433 & 0.139523 & 0.151861 \\
$\theta_{a v 3}(\tau)$ & & & & \\
0.01 & 0.629150 & 0.558417 & 0.614129 & 0.629616 \\
0.05 & 0.266202 & 0.245696 & 0.260075 & 0.262252 \\
0.10 & 0.170676 & 0.174214 & 0.172173 & 0.168900 \\
0.20 & 0.128845 & 0.137187 & 0.131623 & 0.129458 \\
0.50 & 0.088956 & 0.088711 & 0.088780 & 0.089489 \\
1.00 & 0.062749 & 0.060108 & 0.062056 & 0.062847 \\
\hline
\end{tabular}




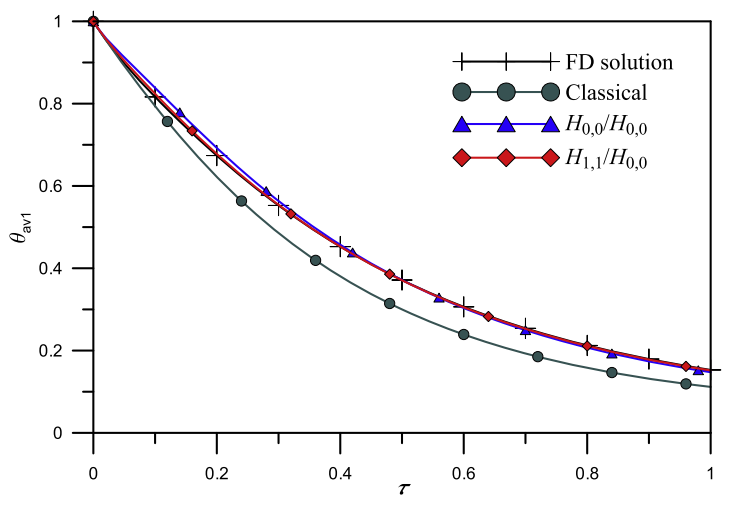

a Dimensionless temperature $\theta_{a v 1}$

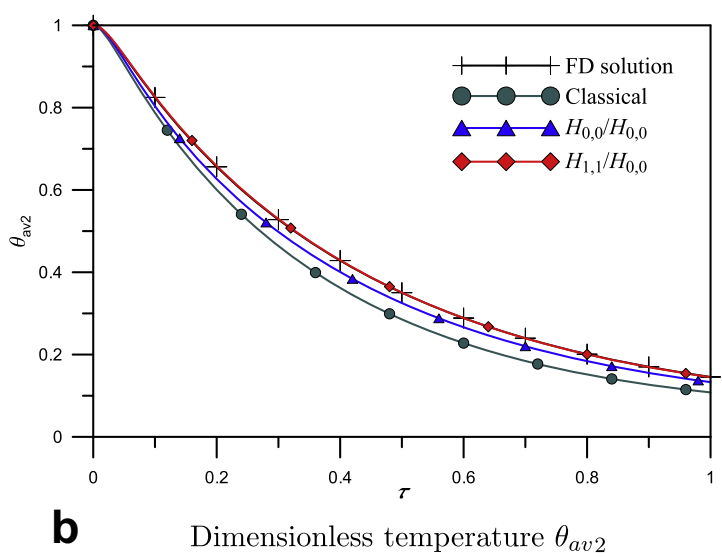

b Dimensionless temperature $\theta_{\text {av2 }}$

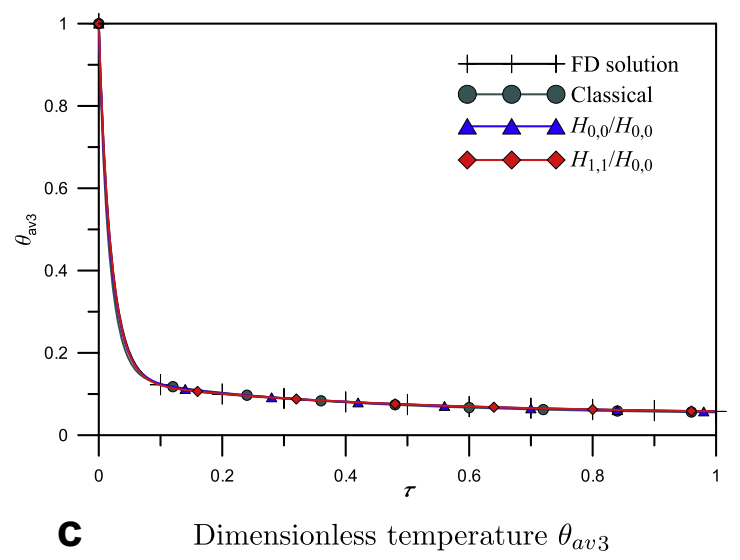

Fig. 2. Dimensionless temperature as a function of dimensionless time for each layer in Case II-3 $\left(B i_{2}=20\right)$.

Let $\delta_{i}$ represent the dimensionless thickness for each layer, $\delta_{i}=$ $\left(x_{i+1}-x_{i}\right) / L, i=1,2, \ldots, M$, where the reference length $L$ is chosen as the total thickness of composite slabs, $x_{m+1}-x_{1}$, hence we have

$\sum_{i=1}^{M} \delta_{i}=1$

The reference thermal conductivity and diffusivity can be taken as those of any layer, say, the second one, then $k_{r e f}=k_{2}$ and $\alpha_{\text {ref }}=\alpha_{2}$. It can be seen that the problem is governed by the following dimensionless parameters, $B i_{1}, B i_{2}, N_{r c}, R c_{i}^{*}, \lambda_{i,} \kappa_{i}, \delta_{i}, \theta_{m}$ and $\theta_{a}, i=1,2, \ldots, M$. The radiation-conduction parameter, $N_{r c}$ that governs the radiative cooling, is conceptually analog to the Biot numbers, $B i_{1}$ and $B i_{2}$, which are the governing parameters for an equivalent transient convective cooling.

\section{Lumped models}

Let us introduce the spatially averaged dimensionless temperature of the $i$-th layer as follows

$\theta_{a v i}(\tau)=\frac{1}{\eta_{i+1}-\eta_{i}} \int_{\eta_{i}}^{\eta_{i+1}} \theta_{i}(\eta, \tau) \mathrm{d} \eta, \quad i=1,2, \ldots, M$.

Operate Eqs. (9) by $\frac{1}{\eta_{i+1}-\eta_{i}} \int_{\eta_{i}}^{\eta_{i+1}} \mathrm{~d} \eta$ and using the definition of average temperatures, Eqs. (17), we have

$\frac{\mathrm{d} \theta_{a v i}(\tau)}{\mathrm{d} \tau}=\frac{\kappa_{i}}{\eta_{i+1}-\eta_{i}}\left(\left.\frac{\partial \theta_{i}}{\partial \eta}\right|_{\eta=\eta_{i+1}}-\left.\frac{\partial \theta_{i}}{\partial \eta}\right|_{\eta=\eta_{i}}\right), i=1,2, \ldots, M$.
Eqs. (18) are an equivalent integro-differential formulation of the original mathematical model, Eqs. (9), with no approximation involved.

Supposing that the temperature gradient is sufficiently smooth over each individual layer, the classical lumped system analysis (CLSA) is based on the assumption that the boundary temperatures can be reasonably well approximated by the average temperature, as

$\left.\theta_{i}(\eta, \tau)\right|_{\eta=\eta_{i}} \cong \theta_{a v i}(\tau), \quad i=1,2, \ldots, M$,

$\left.\theta_{i}(\eta, \tau)\right|_{\eta=\eta_{i+1}} \cong \theta_{\text {avi }}(\tau), \quad i=1,2, \ldots, M$.

For each layer, there are two unknown boundary temperatures, $\left.\theta_{i}\right|_{\eta=\eta_{i}}$ and $\left.\theta_{i}\right|_{\eta=\eta_{i+1}}$ and two unknown heat fluxes, $\partial \theta_{i} /\left.\partial \eta\right|_{\eta=\eta_{i}}$ and $\partial \theta_{i} /\left.\partial \eta\right|_{\eta=\eta_{i+1}}$. Now, we have $2 M$ equations provided by Eqs. $(19,20)$, $2(M-1)$ equations by Eqs. $(12,13)$ and two equations by Eqs. (10, 11 ), that are $4 M$ equations for $4 M$ unknowns which are solved to give the sought relations between the boundary temperatures and heat fluxes and the averaged temperatures in the multi-layer composite slabs. These relations are then used in the Eqs. (18) to yield the classical lumped model which is a closed-form expression with $M$ ordinary differential equations for the averaged temperatures, to be solved with the initial conditions for the averaged temperatures

$\theta_{\text {avi }}(0)=1, \quad i=1,2, \ldots, M$.

In an attempt to improve the approximation of the classical lumped model, we develop higher order lumped models by providing better relations between the boundary temperatures and the average temperatures, based on Hermite-type approximations 


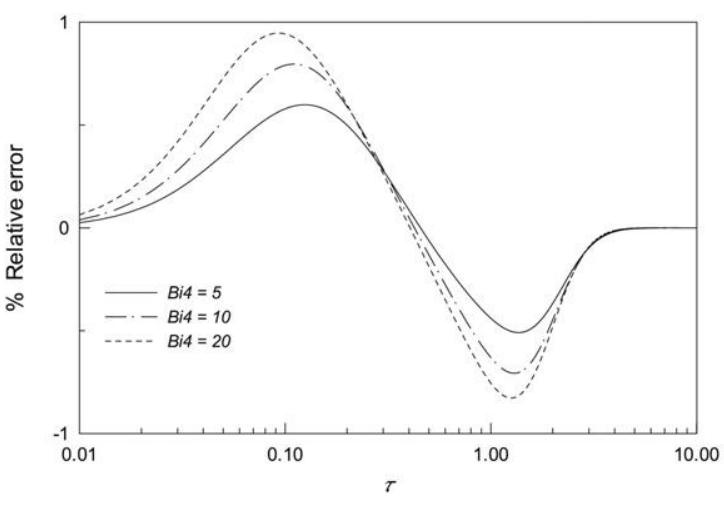

a Relative errors for the first layer

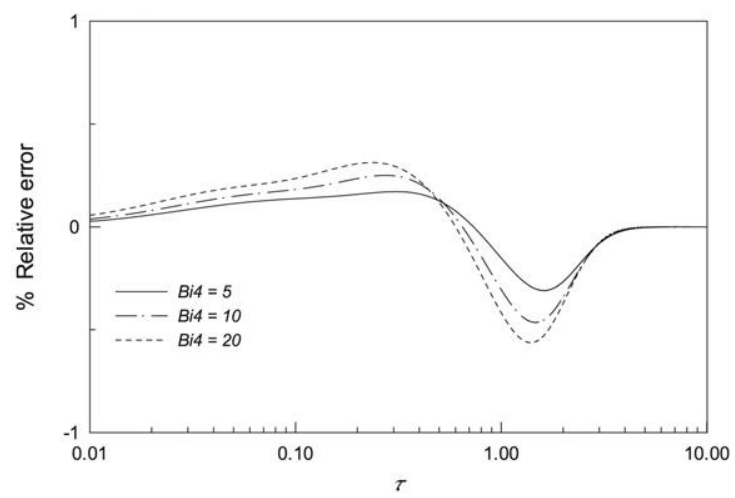

b Relative errors for the second layer

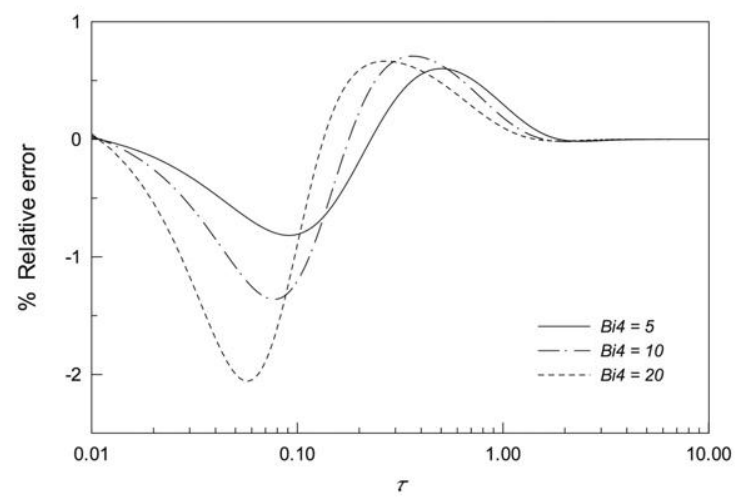

C Relative errors for the third layer

Fig. 3. Temporal variation of the relative errors for the average temperature predicted by the $H_{1,1} / H_{0,0}$ model, for various values of $B i_{2}$.

for integrals that define the average temperatures and the heat fluxes. The general Hermite approximation for an integral, based on the values of the integrand and its derivatives at the integration limits, is written in the following form [33]:

$\int_{a}^{b} y(x) \mathrm{d} x \cong \sum_{\nu=0}^{\alpha} C_{\nu} y^{(\nu)}(a)+\sum_{\nu=0}^{\beta} D_{\nu} y^{(\nu)}(b)$,

where $y(x)$ and its derivatives $y^{(\nu)}(x)$ are defined for all $x \in(a, b)$. It is assumed that the numerical values of $y^{(\nu)}(a)$ for $\nu=0,1, \ldots, \alpha$, and $y^{(\nu)}(b)$ for $\nu=0,1, \ldots, \beta$ are available. The general expression for the $H_{\alpha, \beta}$ approximation is given by

$$
\begin{aligned}
\int_{a}^{b} y(x) \mathrm{d} x= & \sum_{\nu=0}^{\alpha} C_{\nu}(\alpha, \beta) h^{\nu+1} y^{(\nu)}(a) \\
& +\sum_{\nu=0}^{\beta} C_{\nu}(\beta, \alpha)(-1)^{\nu} h^{\nu+1} y^{(\nu)}(b)+O\left(h^{\alpha+\beta+3}\right),
\end{aligned}
$$

where $h=b-a$, and

$C_{\nu}(\alpha, \beta)=\frac{(\alpha+1) !(\alpha+\beta+1-\nu) !}{(\nu+1) !(\alpha-\nu) !(\alpha+\beta+2) !}$.

We first employ the plain trapezoidal rule in the integrals for both average temperatures and average heat fluxes $\left(H_{0,0} / H_{0,0}\right.$ approximation), in the form

$\theta_{\text {avi }}(\tau) \cong \frac{1}{2}\left[\left.\theta_{i}(\eta, \tau)\right|_{\eta=\eta_{i}}+\left.\theta_{i}(\eta, \tau)\right|_{\eta=\eta_{i+1}}\right], \quad i=1,2, \ldots, M$,

$$
\begin{aligned}
\int_{\eta_{i}}^{\eta_{i+1}} \frac{\partial \theta_{i}(\eta, \tau)}{\partial \eta} \mathrm{d} \eta & =\left.\theta_{i}(\eta, \tau)\right|_{\eta=\eta_{i+1}}-\left.\theta_{i}(\eta, \tau)\right|_{\eta=\eta_{i}} \\
& \cong \frac{\eta_{i+1}-\eta_{i}}{2}\left[\left.\frac{\partial \theta_{i}}{\partial \eta}\right|_{\eta=\eta_{i}}+\left.\frac{\partial \theta_{i}}{\partial \eta}\right|_{\eta=\eta_{i+1}}\right], i=1,2, \ldots, M .
\end{aligned}
$$

Analytical solution of the $4 M$ unknowns $\left.\theta_{i}\right|_{\eta=\eta_{i}},\left.\theta_{i}\right|_{\eta=\eta_{i+1}}, \partial \theta_{i} /\left.\partial \eta\right|_{\eta=\eta_{i}}$ and $\partial \theta_{i} /\left.\partial \eta\right|_{\eta=\eta_{i+1}}, \quad i=1,2, \ldots, M$, can be readily obtained from a closed system of Eqs. $(10-13,25,26)$ by using a symbolic computation software such as Mathematica, and then used to close the ordinary differential equations Eqs. (18) for the average temperatures $\theta_{\text {avi }}, i=1,2, \ldots, M$, to be solved with the initial condition Eqs. (21), providing the $H_{0,0} / H_{0,0}$ model.

Then we further improve the lumped model by employing twoside corrected trapezoidal rule in the integral for average temperatures, in the form

$$
\begin{aligned}
\theta_{a v i}(\tau) \cong & \frac{1}{2}\left[\left.\theta_{i}(\eta, \tau)\right|_{\eta=\eta_{i}}+\left.\theta_{i}(\eta, \tau)\right|_{\eta=\eta_{i+1}}\right] \\
& +\frac{\eta_{i+1}-\eta_{i}}{12}\left[\left.\frac{\partial \theta_{i}}{\partial \eta}\right|_{\eta=\eta_{i}}-\left.\frac{\partial \theta_{i}}{\partial \eta}\right|_{\eta=\eta_{i+1}}\right], \quad i=1,2, \ldots, M,
\end{aligned}
$$

while keeping the plain trapezoidal rule in the integral for heat fluxes ( $H_{1,1} / H_{0,0}$ approximation).

Similarly, the boundary temperatures and heat fluxes can be obtained from Eqs. $(10-13,26,27)$ and used to close the ordinary differential equations Eqs. (18) for the average temperatures, to be solved with the initial conditions Eqs. (21), providing the $H_{1,1} / H_{0,0}$ model. 


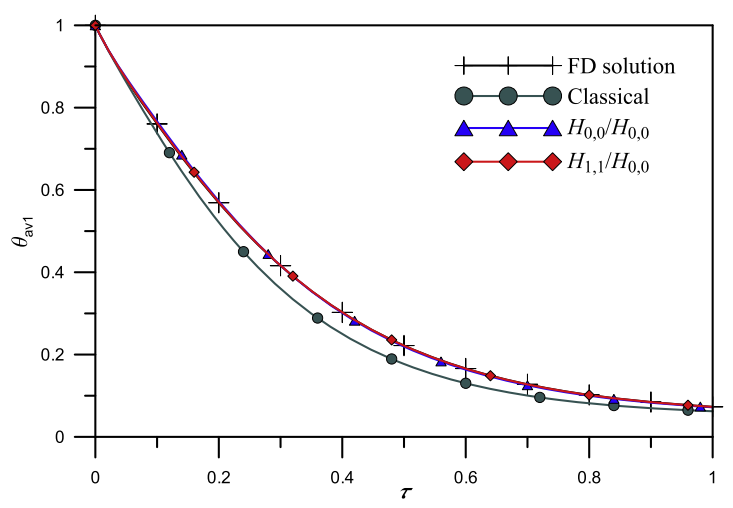

a Dimensionless temperature $\theta_{a v 1}$

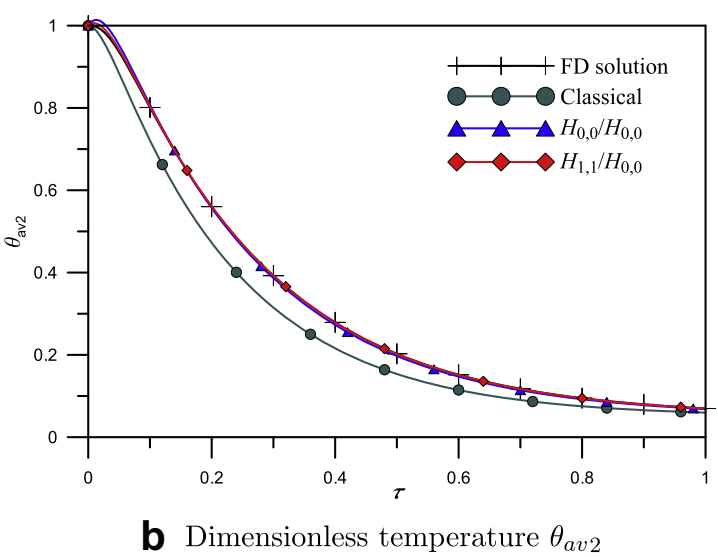

b Dimensionless temperature $\theta_{a v 2}$

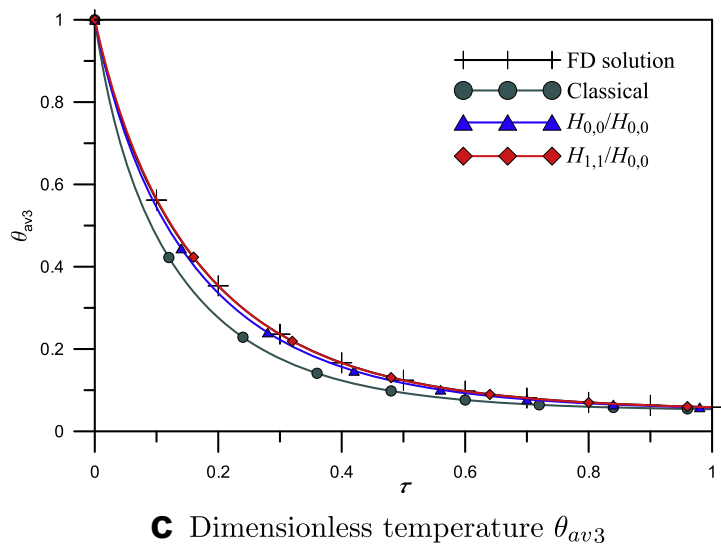

Fig. 4. Dimensionless temperature as a function of dimensionless time for each layer in Case III- $1\left(\delta_{3}=0.7\right)$.

\section{Numerical results and discussions}

Transient heat conduction in three-layer composite slabs with combined convective and radiative cooling is analyzed to illustrate the applicability of the proposed lumped models. The solutions of classical and improved lumped models are presented in tabular and graphical forms in comparison with a reference finite difference solution of the original distributed model, Eqs. (9-14). The initial boundary value problem defined by Eqs. (9-14) is solved by using an implicit finite difference method, with a 101 nodes mesh in spatial discretization for each layer and a dimensionless time step of 0.000001 for all cases. Different values of the Biot number $B i_{2}$, the radiation-conduction parameter $N_{r c}$, dimensionless thermal contact resistances $R c_{1}^{*}$ and $R c_{2}^{*}$, dimensionless thickness $\delta_{3}$ and dimensionless thermal conductivity $\lambda_{3}$ are chosen so as to assess accuracy of the solutions obtained by the lumped models.

To make the expression concise, the examined problems with different cases are defined as follows:

Problem I. $R c_{1}^{*}=0.5, R c_{2}^{*}=0.5, B i_{2}=12, \delta_{1}=0.2, \delta_{2}=0.6$, $\delta_{3}=0.2, \lambda_{3}=10, \kappa_{3}=5$. Case I-1: $N_{r c}=8$; Case I-2: $N_{r c}=32$.

Problem II. $R c_{1}^{*}=0.5, R c_{2}^{*}=0.5, N_{r c}=5, \delta_{1}=0.2, \delta_{2}=0.6$, $\delta_{3}=0.2, \lambda_{3}=10, \kappa_{3}=5$. Case II- $1: B i_{2}=5$; Case II-2: $B i_{2}=10$; Case II-3: $B i_{2}=20$.

Problem III. $R c_{1}^{*}=0.5, R c_{2}^{*}=0.5, B i_{2}=10, N_{r c}=5, \lambda_{3}=10$, $\kappa_{3}=5$. Case III- 1 : $\delta_{1}=0.15, \delta_{2}=0.15, \delta_{3}=0.7$; Case III-2: $\delta_{1}=0.05, \delta_{2}=0.05, \delta_{3}=0.9$.

Problem IV. $R c_{1}^{*}=0.5, R c_{2}^{*}=0.5, B i_{2}=10, N_{r c}=5, \delta_{1}=0.2$, $\delta_{2}=0.6, \delta_{3}=0.2$. Case IV-1: $\lambda_{3}=2, \kappa_{3}=1$; Case IV-2: $\lambda_{3}=1$, $\kappa_{3}=0.5$.
Problem V. $B i_{2}=10, N_{r c}=5, \delta_{1}=0.2, \delta_{2}=0.6, \delta_{3}=0.2$, $\lambda_{3}=10, \kappa_{3}=5$. Case V-1: $R c_{1}^{*}=0.1, R c_{2}^{*}=0.1$; Case V-2: $R c_{1}^{*}=10^{-7}, R c_{2}^{*}=10^{-7}$.

For all the problems tested, $B i_{1}=1, \lambda_{1}=10, \lambda_{2}=1, \kappa_{1}=5$, $\kappa_{2}=1, \theta_{m}=0.05, \theta_{a}=0.05$, which are kept fixed in the following calculations.

Tables 1 and 2 present comparisons between the dimensionless average temperatures obtained by lumped models and the reference finite difference solution of the original distributed parameter model for each layer at the different dimensionless time, whose parameters are defined, respectively, by Case I- 1 and Case I- 2 in Problem I. As can be calculated at $\tau=1.00$ in Case I-1, the classical lumped model gives errors of $-25.137 \%,-24.080 \%$ and $-4.153 \%$ for each layer, while the $H_{0,0} / H_{0,0}$ model gives errors of $-3.360 \%,-8.426 \%$ and $-1.102 \%$, and the $H_{1,1} / H_{0,0}$ model yields $-0.669 \%,-0.339 \%$ and $0.157 \%$, respectively.

Fig. 2 presents the behaviors of the lumped models for Case II-3. It can be seen that the higher order lumped model $\left(H_{1,1} / H_{0,0}\right.$ approximation) presents good agreement with the reference finite difference solution for values of $B i_{2}$ as high as 20 and $N_{r c}$ as high as 5 . The overall error patterns for the proposed improved lumped model $H_{1,1} / H_{0,0}$ as a function of the logarithm of the dimensionless time are shown in Fig. 3. We can observe that for each case the error rises to maximum in early time, decays to zero in intermediate times, then reaches to the peak value in the opposite direction and finally diminishes to zero again in long times. The absolute values of the maximum relative errors for Case II-3 are around $0.95 \%, 0.55 \%$ and $2.05 \%$ respectively for each layer.

In Problem III, different values of the dimensionless thickness $\delta_{3}$ are chosen in order to investigate the dimensionless temperature as a function of dimensionless time solved by the lumped models. In 


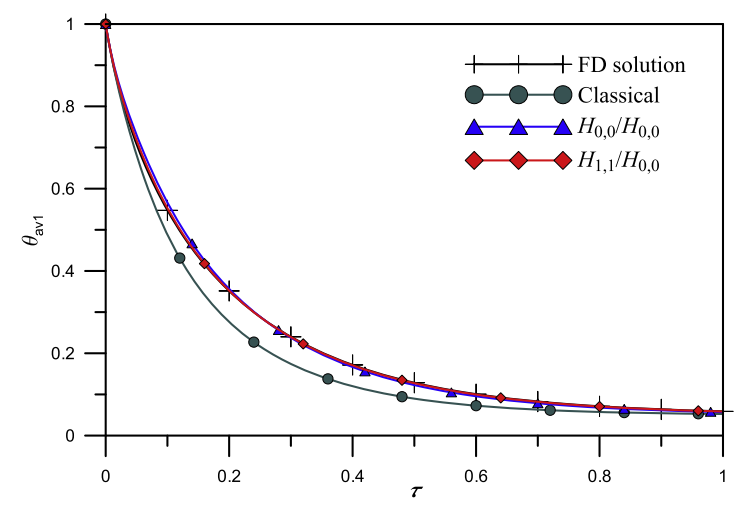

a Dimensionless temperature $\theta_{a v 1}$

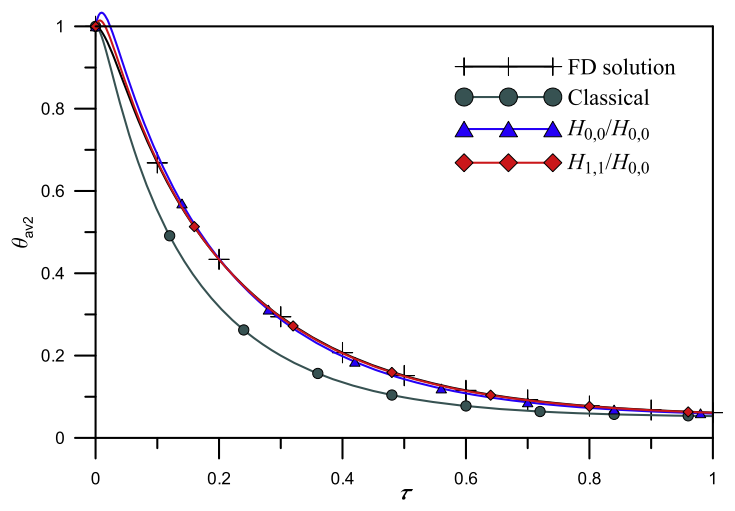

b Dimensionless temperature $\theta_{a v 2}$

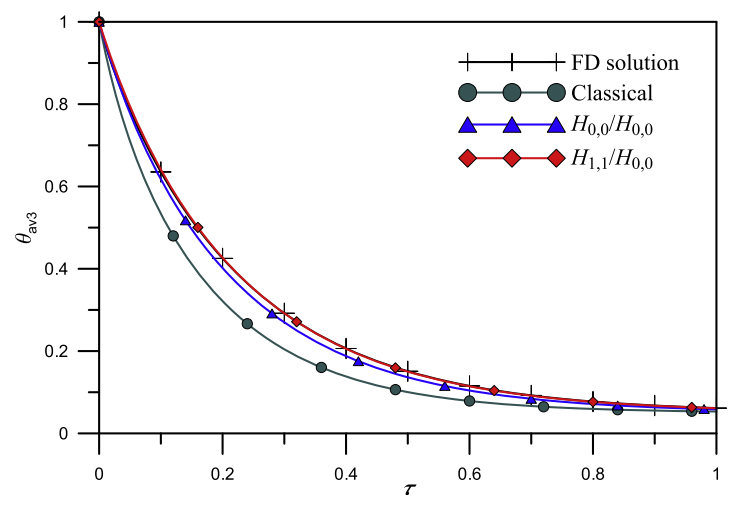

C Dimensionless temperature $\theta_{\text {av } 3}$

Fig. 5. Dimensionless temperature as a function of dimensionless time for each layer in Case III-2 $\left(\delta_{3}=0.9\right)$.

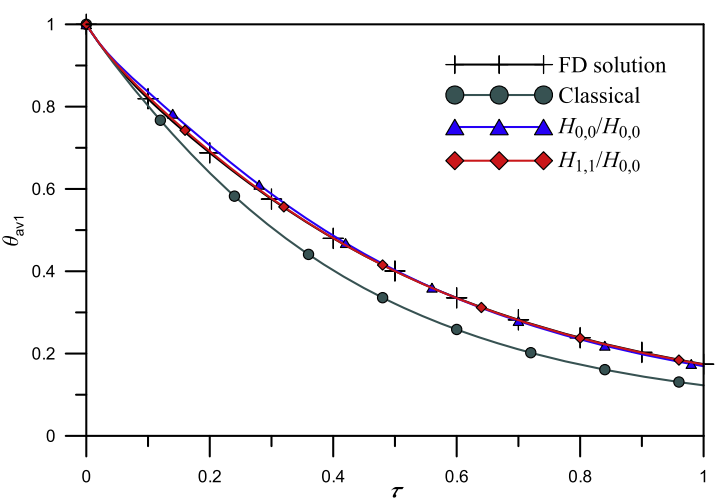

a Dimensionless temperature $\theta_{a v 1}$

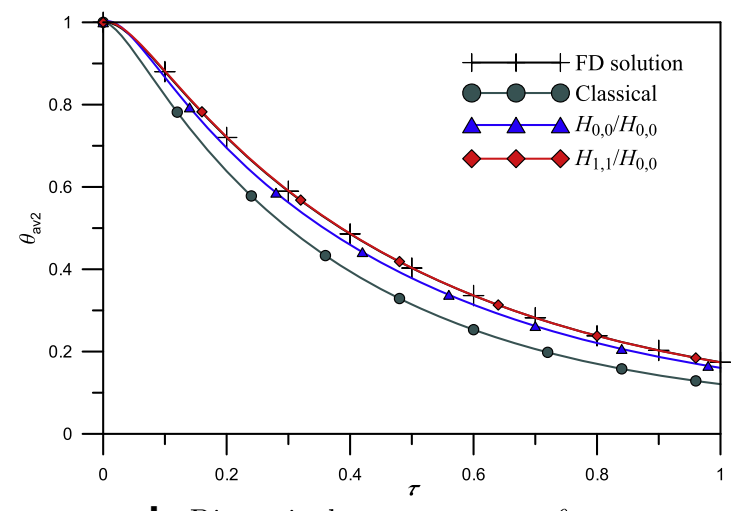

b Dimensionless temperature $\theta_{a v 2}$

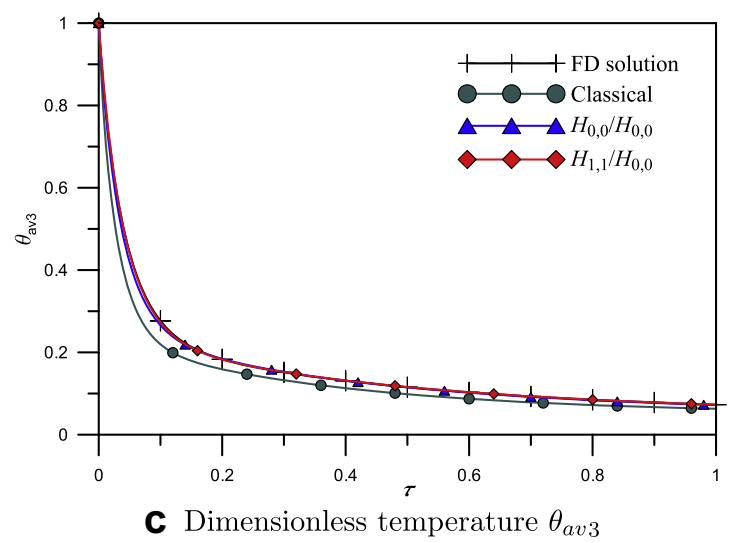

Fig. 6. Dimensionless temperature as a function of dimensionless time for each layer in Case IV-1 $\left(\lambda_{3}=2, \kappa_{3}=1\right)$. 


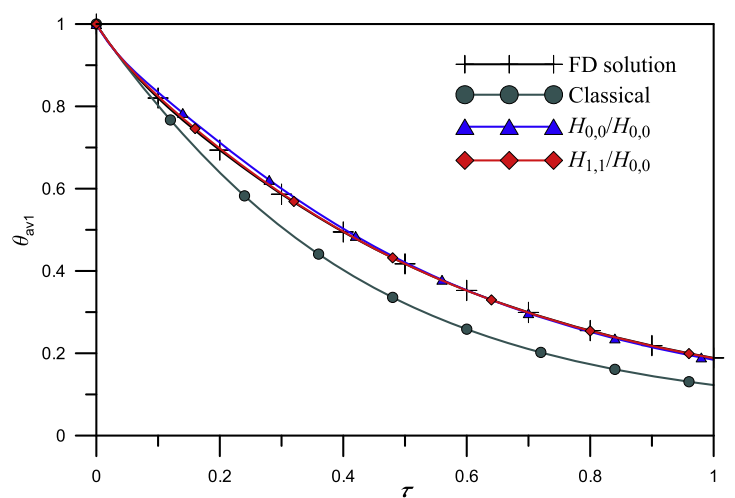

a Dimensionless temperature $\theta_{a v 1}$

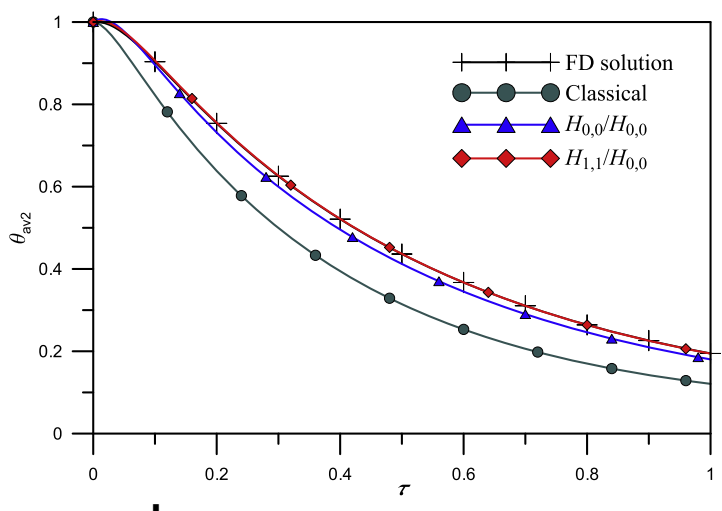

b Dimensionless temperature $\theta_{\text {av } 2}$

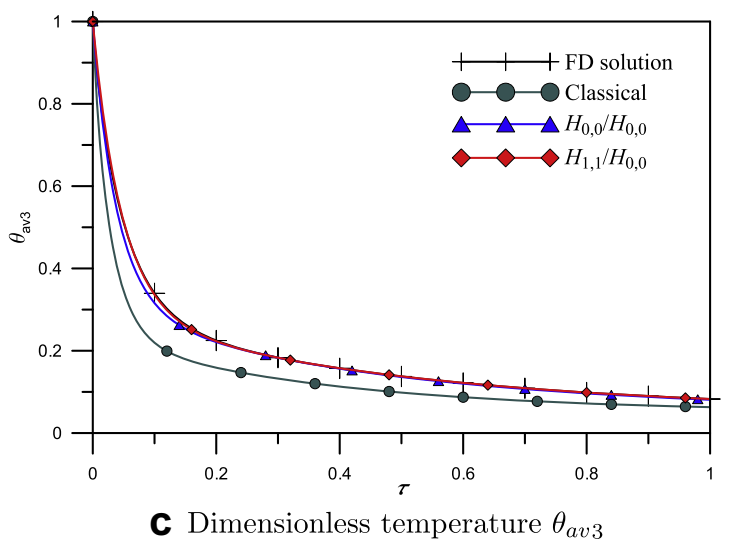

Fig. 7. Dimensionless temperature as a function of dimensionless time for each layer in Case IV-2 $\left(\lambda_{3}=1, \kappa_{3}=0.5\right)$.

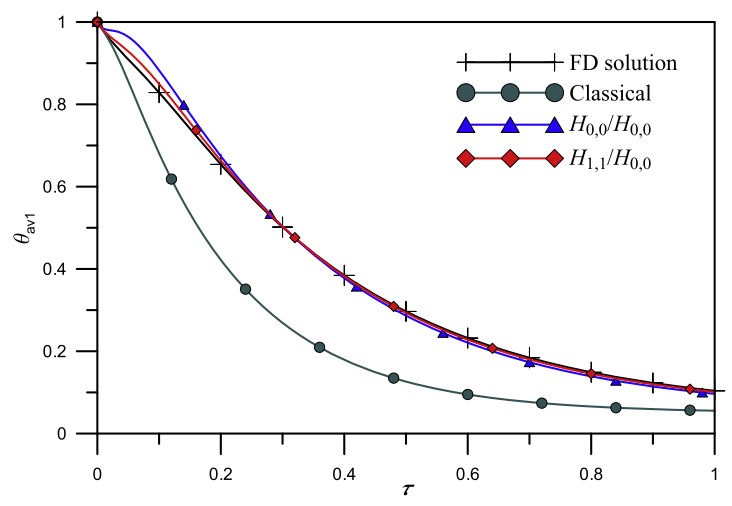

a Dimensionless temperature $\theta_{a v 1}$

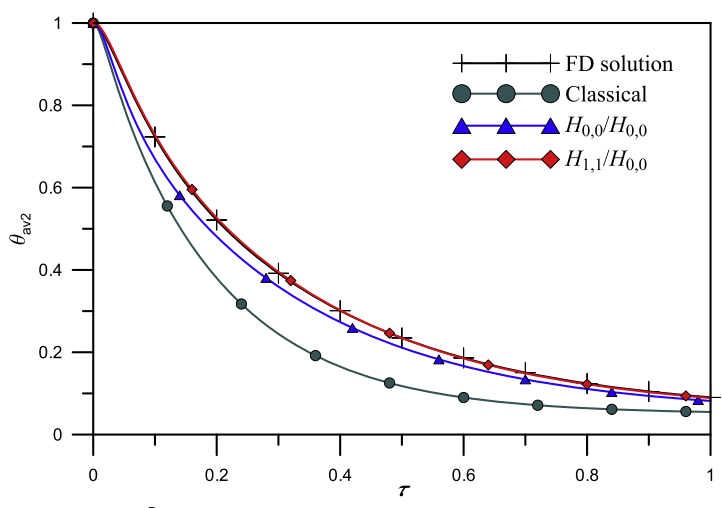

b Dimensionless temperature $\theta_{\text {av } 2}$

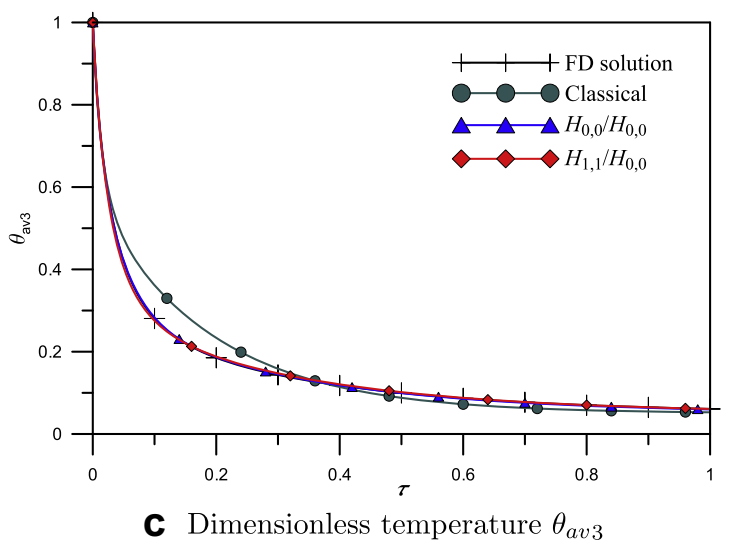

Fig. 8. Dimensionless temperature as a function of dimensionless time for each layer in Case V-1 $\left(R c_{1}^{*}=0.1, R c_{2}^{*}=0.1\right)$. 


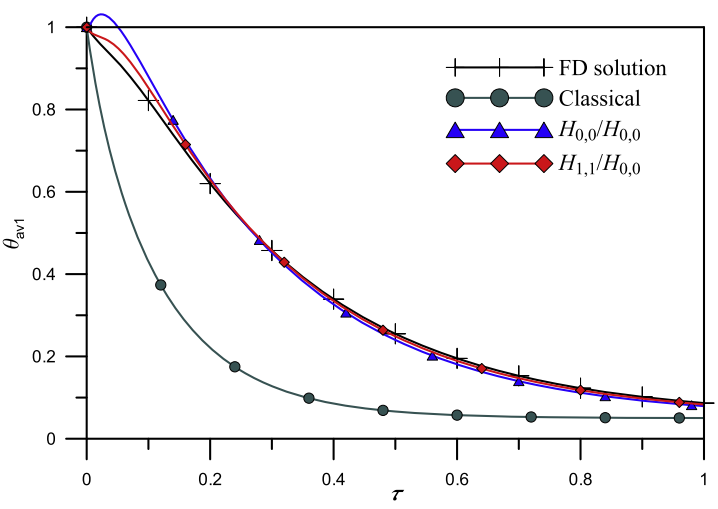

a Dimensionless temperature $\theta_{a v 1}$

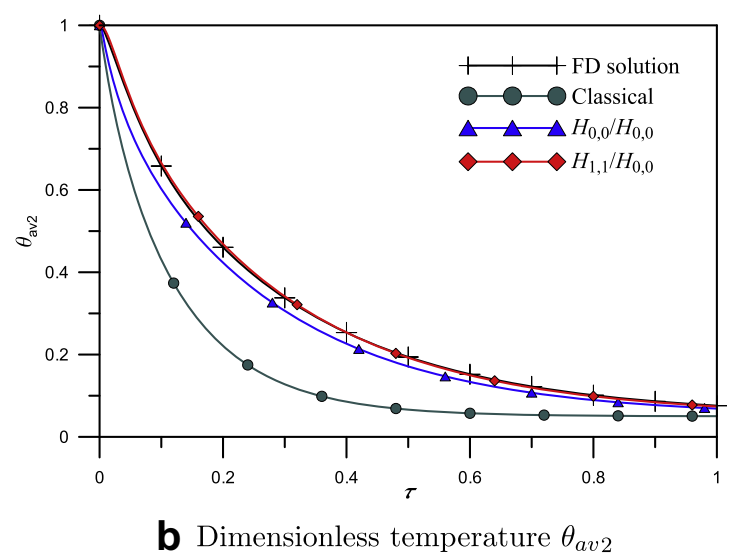

b Dimensionless temperature $\theta_{a v 2}$

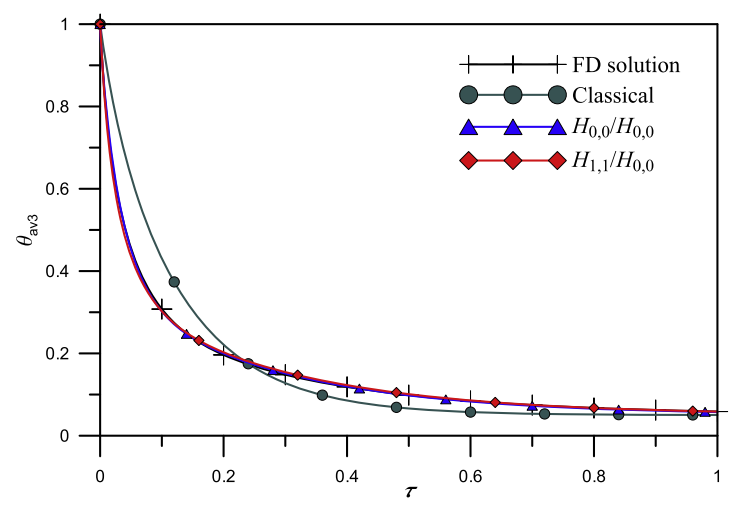

C Dimensionless temperature $\theta_{a v 3}$

Fig. 9. Dimensionless temperature as a function of dimensionless time for each layer in Case V-2 $\left(R c_{1}^{*}=10^{-7}, R c_{2}^{*}=10^{-7}\right)$.

each case, the value of the dimensionless thickness $\delta_{1}$ is assumed to be same as the value of $\delta_{2}$. As shown in Figs. 4 and 5, although all the lumped models predict the correct value of the steady-state temperature for all the three layers, the classical lumped model gives larger relative errors for the whole time interval up to $\tau=1$, while the improved lumped model $H_{0,0} / H_{0,0}$ presents smaller relative errors, and the $H_{1,1} / H_{0,0}$ model yields a good agreement with the finite difference solution. It is important to observe that as the value of the dimensionless thickness $\delta_{3}$ increases, the improvement offered by the proposed models becomes more evident for the third layer compared with the above cases.

The numerical results of transient history for Case IV-1 and Case IV-2 are shown in Figs. 6 and 7. We can observe again that the averaged dimensionless temperature obtained by the improved lumped parameter formulation $\left(H_{1,1} / H_{0,0}\right.$ approximation) is excellent agreement with the finite difference solution. It should be also noted that with the value of the dimensionless thermal conductivity $\lambda_{3}$ decreasing (the dimensionless thermal diffusivity $\kappa_{3}$ is directly proportional to $\lambda_{3}$ ), the improvement offered by the proposed models becomes more evident.

The next group of cases in Problem $V$ are performed with the decreasing of the values of $R c_{1}^{*}$ and $R c_{2}^{*}$, as shown in Figs. 8 and 9. Note that when the values of $R c_{1}^{*}$ and $R c_{2}^{*}$ reach to infinitesimal, imperfect contact interface boundary condition will convert to its special case that is perfect thermal contact condition at the interfaces. In Case $\mathrm{V}-2, R c_{1}^{*}=10^{-7}$ and $R c_{2}^{*}=10^{-7}$ are utilized to simulate approximately the behaviors of the lumped models with perfect thermal contact conditions. Again, we can see that the prediction from the $H_{1,1} / H_{0,0}$ model appears clearly closer to the FD solution than the results from the $H_{0,0} / H_{0,0}$ one, while the classical lumped model fails to give accurate results.

Finally, the higher order $\left(H_{1,1} / H_{0,0}\right)$ model is employed to analyze the transient combined convective and radiative cooling of steel-concrete-steel sandwich plates, which present a common structure in engineering applications such as advanced nuclear reactors. The parameters used in this practical case are given in Table 3. The first and third layers are made of chrome steel $(5 \% \mathrm{Cr})$, while the interlayer material is dry concrete. The corresponding Biot numbers and radiation-conduction parameter can be calculated from Eqs. (15) that $B i_{1}=8, B i_{2}=50$ and $N_{r c} \approx 3$. Fig. 10 shows the average temperature as a function of time for each layer of the sandwich plates. It can be clearly observed that before $t=50 \mathrm{~s}$, the temperature of the third layer reduces to $400 \mathrm{~K}$, while the first layer needs more than $600 \mathrm{~s}$ to reach the same temperature. The cooling of the concrete interlayer is slowest due to its higher thermal capacity and lower thermal conductivity.

Table 3

Parameters of steel-concrete-steel sandwich plates.

\begin{tabular}{llllll}
\hline$k_{1}$ & $(\mathrm{~W} / \mathrm{m} \mathrm{K})$ & 40 & $\epsilon$ & & 0.85 \\
$\rho_{1}$ & $\left(\mathrm{~kg} / \mathrm{m}^{3}\right)$ & 7833 & $R c_{1}$ & $\left(\mathrm{~m}^{2} \mathrm{~K} / \mathrm{W}\right)$ & 0.005 \\
$c_{p 1}$ & $(\mathrm{~J} / \mathrm{kg} \mathrm{K})$ & 0.46 & $R c_{2}$ & $\left(\mathrm{~m}^{2} \mathrm{~K} / \mathrm{W}\right)$ & 0.005 \\
$k_{2}$ & $(\mathrm{~W} / \mathrm{m} \mathrm{K}))$ & 1.28 & $h_{1}$ & $\left(\mathrm{~W} / \mathrm{m}^{2} \mathrm{~K}\right)$ & 204.8 \\
$\rho_{2}$ & $\left(\mathrm{~kg} / \mathrm{m}^{3}\right)$ & 2200 & $h_{2}$ & $\left(\mathrm{~W} / \mathrm{m}^{2} \mathrm{~K}\right)$ & 1280 \\
$c_{p 2}$ & $(\mathrm{~J} / \mathrm{kg} \mathrm{K})$ & 0.88 & $T_{m}$ & $(\mathrm{~K})$ & 298 \\
$k_{3}$ & $(\mathrm{~W} / \mathrm{m} \mathrm{K}))$ & 40 & $T_{f}$ & $(\mathrm{~K})$ & 298 \\
$\rho_{3}$ & $\left(\mathrm{~kg} / \mathrm{m}^{3}\right)$ & 7833 & $T_{s}$ & $(\mathrm{~K})$ & 298 \\
$c_{p 3}$ & $(\mathrm{~J} / \mathrm{kg} \mathrm{K})$ & 0.46 & $T_{0}$ & $(\mathrm{~K})$ & 1173 \\
\hline
\end{tabular}




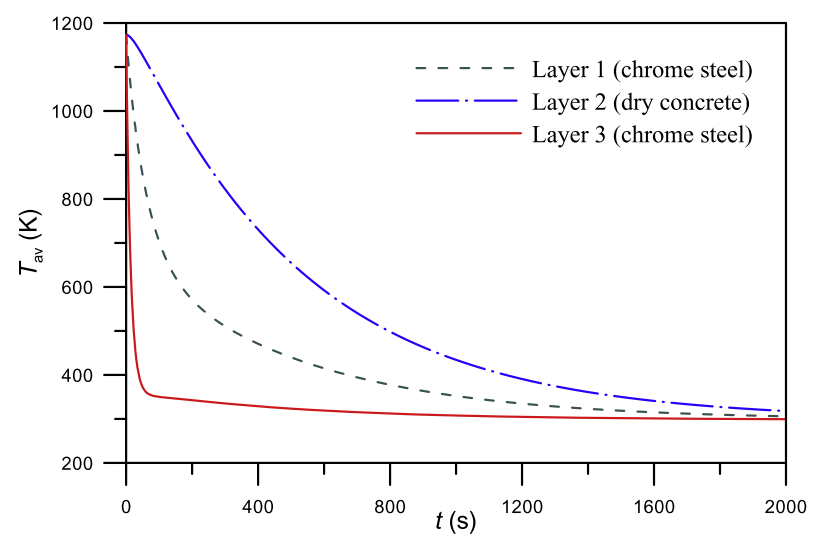

Fig. 10. Average temperature as a function of time for each layer of steel-concrete-steel sandwich plates.

\section{Conclusions}

Obtained through two-point Hermite approximations for integrals, improved lumped parameter models were developed for the transient heat conduction in multi-layer composite slabs subjected to combined convective and radiative cooling. Three-layer composite slabs were analyzed to illustrate the applicability of the proposed lumped models, with respect to different values of the Biot numbers, the radiation-conduction parameter, the dimensionless thickness, and the dimensionless thermal conductivity. It was shown by comparison with numerical solution of the original distributed parameter model that the higher order lumped model $\left(H_{1,1} / H_{0,0}\right.$ approximation) yielded significant improvement of average temperature prediction over the classical lumped model, for radiation-conduction parameter as high as 32 , and Biot number as high as 20. It was observed that the error rises to maximum in early time, decays to zero in intermediate times, then reaches to the peak value in the opposite direction and finally diminishes to zero again in long times. It was found that as value of the dimensionless thickness $\delta_{3}$ increases or value of the dimensionless thermal conductivity $\lambda_{3}$ decreases, the improvement offered by the proposed models becomes more evident. For perfect thermal contact condition at the interfaces, it was found that the prediction from the $H_{1,1} / H_{0.0}$ model has a better agreement with the FD solution than the results from the $H_{0,0} / H_{0,0}$ one, while the classical lumped model fails to give accurate results. The analysis of transient combined convective and radiative cooling of steel-concrete-steel sandwich plates illustrates the potential applications of the proposed improved lumped model in practical engineering problems.

\section{Acknowledgements}

The authors acknowledge gratefully the financial support provided by CNPq, CAPES and FAPERJ of Brazil for their research work. C. An acknowledges gratefully the financial support provided by the China Scholarship Council.

\section{References}

[1] H. Asan, Investigation of wall's optimum insulation position from maximum time lag and minimum decrement factor point of view, Energ. Buildings 32 (2) (2000) 197-203.

[2] H.T. Ozkahraman, A. Bolatturk, The use of tuff stone cladding in buildings for energy conservation, Constr. Build. Mater 20 (7) (2006) 435-440.
[3] M. Bouzidi, P. Duhamel, Non-stationary heat conduction in composite slabs with coupling. Application to enclosures: numerical computation of the analytical solution, Comput. Math. Appl. 11 (10) (1985) 1043-1055.

[4] A. Charette, R.T. Bui, G. Simard, The effect of stringent boundary-conditions on the solution of a transient heat-conduction problem, Math. Comput. Simulat 27 (1) (1985) 47-60.

[5] M. Blanc, M. Touratier, A constrained discrete layer model for heat conduction in laminated composites, Comput. Struct. 83 (2005) 1705-1718.

[6] G. Oturanç, A.Z. Sahin, Eigenvalue analysis of temperature distribution in composite walls, Int. J. Energ. Res. 25 (13) (2001) 1189-1196.

[7] M. Spinnler, E.R.F. Winter, R. Viskanta, Studies on high-temperature multilayer thermal insulations, Int. J. Heat Mass Tran 47 (6-7) (2004) 1305-1312.

[8] P. Li, H. Cheng, Thermal analysis and performance study for multilayer perforated insulation material used in space, Appl. Therm. Eng. 26 (16) (2006) 2020-2026.

[9] F. de Monte, Transient heat conduction in one-dimensional composite slab. a 'natural' analytic approach, Int. J. Heat Mass Tran 43 (19) (2000) 3607-3619.

[10] F. de Monte, An analytic approach to the unsteady heat conduction processes in one-dimensional composite media, Int. J. Heat Mass Tran 45 (6) (2002) 1333-1343.

[11] Y.M. Chen, S.W. Wang, Frequency-domain regression method for estimating ctf models of building multilayer constructions, Appl. Math. Model. 25 (7) (2001) 579-592.

[12] S.W. Wang, Y.M. Chen, A simple procedure for calculating thermal response factors and conduction transfer functions for multilayer walls, Appl. Therm. Eng. 22 (2002) 333-338.

[13] K.A. Antonopoulos, C. Tzivanidis, M. Vrachopoulos, Using orthogonal expansion of functions over multilayer walls for calculating the layer thermal properties, Appl. Therm. Eng. 17 (2) (1997) 193-201.

[14] B. Sundén, Transient heat-conduction in a composite slab by a time-varying incident heat-flux combined with convective and radiative cooling, Int. Commun. Heat Mass 13 (1986) 515-522.

[15] J.R. Miller, P.M. Weaver, Temperature profiles in composite plates subject to time-dependent complex boundary conditions, Compos. Struct. 59 (2) (2003) 267-278.

[16] K.A. Antonopoulos, E.P. Koronaki, Envelope and indoor thermal capacitance of buildings, Appl. Therm. Eng. 19 (1999) 743-756.

[17] K.A. Antonopoulos, E.P. Koronaki, Thermal parameter components of building envelope, Appl. Therm. Eng. 20 (2000) 1193-1211.

[18] P.E. Ergatis, P.G. Massouros, G.C. Athanasouli, G.P. Massouros, Time-dependent heat transfer coefficient of a wall, Int. J. Energ. Res. 27 (2003) 795-811.

[19] A.C.S. Estrada-Flores, D. Cleland, Prediction of the dynamic thermal behaviour of walls for refrigerated rooms using lumped and distributed parameter models, Int. J. Refrig 24 (2001) 272-284.

[20] P.T. Tsilingiris, On the thermal time constant of structural walls, Appl. Therm. Eng. 24 (5-6) (2004) 743-757.

[21] F. Alhama, J. Zueco, Application of a lumped model to solids with linearly temperature-dependent thermal conductivity, Appl. Math. Model. 31 (2) (2007) 302-310.

[22] R.M. Cotta, M.D. Mikhailov (Eds.), Heat Conduction - Lumped Analysis, Integral Transforms, Symbolic Computation, John Wiley \& Sons, Chichester, England, 1997.

[23] J. Su, Improved lumped models for asymmetric cooling of a long slab by heat convection, Int. Commun. Heat Mass 28 (2001) 973-983.

[24] J. Su, Improved lumped models for transient radioactive cooling of a spherical body, Int. Commun. Heat Mass 31 (2004) 85-94.

[25] O. Bautista, F. Mendez, I. Campos, Transient heat conduction in a solid slab using multiple-scale analysis, Heat Mass Transf 42 (2005) 150-157.

[26] H. Sadat, A general lumped model for transient heat conduction in onedimensional geometries, Appl. Therm. Eng. 25 (2005) 567-576.

[27] H. Sadat, A second order model for transient heat conduction in a slab with convective boundary conditions, Appl. Therm. Eng. 26 (2006) 962-965.

[28] C.R. Regis, R.M. Cotta, J. Su, Improved lumped analysis of transient heat conduction in a nuclear fuel rod, Int. Commun. Heat Mass 27 (2000) 357-366.

[29] J. Su, R.M. Cotta, Improved lumped parameter formulation for simplified lwr thermohydraulic analysis, Ann. Nucl. Energy 28 (2001) 1019-1031.

[30] N.J. Ruperti Jr., C.V. Falkenberg, R.M. Cotta, J. Su, Engineering analysis of ablative thermal protection for atmospheric reentry: improved lumped formulations and symbolic-numerical computation, Heat Transfer Eng. 25 (2004) 101-111.

[31] G. Su, Z. Tan, J. Su, Improved lumped models for transient heat conduction in a slab with temperature-dependent thermal conductivity, Appl. Math. Model. 33 (1) (2009) 274-283.

[32] Z. Tan, G. Su, J. Su, Improved lumped models for combined convective and radiative cooling of a wall, Appl. Therm. Eng. 29 (11-12) (2009) 2439-2443.

[33] J. Mennig, T. Auerbach, W. Hälg, Two point hermite approximation for the solution of linear initial value and boundary value problems, Comput. Method Appl. M. 39 (1983) 199-224. 